\title{
émulations
}

\section{Frontières et marges de la parenté}

\author{
Agnès Fine
}

Émulations - Revue de sciences sociales

2019, n 32, «Aux frontières de la parenté. Un éclairage par les marges ».

\section{Article disponible à l'adresse suivante}

https://ojs.uclouvain.be/index.php/emulations/article/view/fine

\section{Pour citer cet article}

Agnès Fine, «Frontières et marges de la parenté », Émulations, $n^{\circ} 32$, Mise en ligne le 20 avril 2020.

DOI : 10.14428/emulations.032.09

Distribution électronique : Université catholique de Louvain (Belgique) : ojs.uclouvain.be

(c) Cet article est mis à disposition selon les termes de la Licence Creative Commons Attribution, Pas d'Utilisation Commerciale 4.0 International. http://creativecommons.org/licenses/by-nc/4.0/

Éditeur : Émulations - Revue de sciences sociales / Presses universitaires de Louvain https://ojs.uclouvain.be/index.php/emulations

ISSN électronique : 1784-5734

PUL PRESSES

UNIVERSITAIRES 


\section{Frontières et marges de la parenté}

Agnès Fine ${ }^{1}$

Ce dossier comprend cinq textes de disciplines différentes, sociologie, anthropologie, travail social et histoire, dans lesquels ont été mises à l'épreuve les notions de « frontière » et de «marge » dans le domaine de la parenté. Excellente initiative que de prolonger le dialogue entre ces quatre disciplines, un dialogue qui a été particulièrement fructueux dans le domaine de la parenté des sociétés européennes passées et présentes depuis les années 1970. En bonne anthropologue comparatiste, j'ai essayé d'identifier les points communs et/ou les divergences de ces textes pour faire ressortir leurs apports et éventuellement ouvrir des pistes nouvelles. L’exercice s'est révélé ardu, car si la problématique générale semble la même, chacun d'eux s'attache à un domaine particulier du champ de la parenté, l'alliance pour deux d'entre eux, la filiation pour les trois autres. En outre, il est question de sociétés parfois difficilement comparables parce que très éloignées dans l'espace ou dans le temps. Enfin, à ces difficultés, s'en ajoute une dernière plus importante : l'application de la notion de frontière à des espaces très variés ainsi que la polysémie de celle de marge dont il est fait un usage très différent selon les textes.

\section{Frontière spatiale dans l'alliance et relation entre les sexes}

Il est question de frontière spatiale et de rapport entre les sexes au sein du lien conjugal dans les textes de Delphine Manetta et d'Anne Lavanchy, de manière explicite dans le premier qui traite de l'alliance dans quelques villages jàana du Burkina Faso, de manière implicite dans le second, qui s'intéresse au mariage entre Suisse et étranger. S'inspirant des anthropologues qui analysent la parenté en terme spatial, D. Manetta analyse les conditions et les effets de la mobilité féminine liée aux règles de l'alliance qui, dans la région étudiée, imposent une résidence patri-virilocale. Cette mobilité est perçue comme ambivalente, positive en ce qu'elle permet la reproduction de la vie, mais négative en ce qu'elle conduirait aussi très souvent les hommes à la mort. C'est pourquoi il convient que les hommes immobiles contrôlent la mobilité des femmes mariées, que ces dernières s'immobilisent peu à peu à l'intérieur de leurs maisons, leur mobilité constituant un danger. Ici la relation structurellement conflictuelle entre les sexes induite par les règles d'alliance s'exprime en clef d'espace.

C'est aussi la frontière spatiale (et juridique) à l'intérieur même du lien conjugal qui est au cœur de l'objet de recherche d'A. Lavanchy. L'auteure analyse les modalités concrètes de l'examen administratif permettant l'accès à la nationalité suisse par le mariage. Une forme d'accès relativement peu étudiée auparavant, et pourtant impor-

\footnotetext{
${ }^{1}$ Université Toulouse-Jean Jaurès, LISST-CAS, France.
} 
tante dans un contexte où la Suisse ne connaît pas le droit du sol' ${ }^{2}$. La frontière suisse est bien défendue par le personnel étatique censé débusquer les mariages blancs. Tout l'enjeu de son travail est d'évaluer le «bon mariage » mixte, celui qui correspond aux normes dominantes dans la société globale, un mariage d'amour. L'enquête met au jour un élément inattendu : l'asymétrie entre les sexes qu'exprime la notion de « mariage gris » inventée par le personnel administratif, à savoir un mariage unissant une femme suisse sincèrement amoureuse à un conjoint étranger calculateur et intéressé. Cette notion révèle les représentations dominantes sur les femmes vues comme des êtres sentimentaux et vulnérables, et sur les hommes étrangers perçus comme un danger pour la société suisse. L'auteure les rapproche à juste titre du droit suisse de la nationalité qui, s’il a été aboli récemment sur ce point, est révélateur des conditions sexuées d'appartenance à la nation. À son mariage avec un Suisse, l'épouse étrangère perdait sa nationalité d'origine, alors que l'épouse suisse d'un homme étranger perdait immédiatement sa nationalité pour adopter celle de son époux. On aimerait en savoir plus sur ces frontières-là qui se jouent entre les sexes, à l'intérieur même de l'alliance, ce qui éclairerait en retour le contenu de l'appartenance nationale dont le caractère genré reste encore en partie inaperçu.

Notons que les deux textes traitant de l'alliance révèlent, avec la notion de frontière spatiale, l'asymétrie entre les sexes tant au Burkina Faso qu'en Suisse. Ce constat suggère que penser la mobilité d'un des conjoints induite par l'alliance constitue une piste fructueuse à explorer si l'on veut saisir les relations de pouvoir d'un sexe sur l'autre. Ne faudrait-il pas reprendre à nouveaux frais, dans une perspective de genre, la question des règles de résidence après le mariage, chapitre obligé des études anthropologiques de la parenté dont on ne tire peut-être pas tous les enseignements?

\section{La frontière entre les vivants et les morts}

Lucie Jégat s'est donné pour objet d'explorer une des frontières majeures des sociétés humaines, celle qui sépare les vivants et les morts. Saluons la pertinence pour l'étude de la parenté de cet objet de recherche peu exploré. L'auteure met à l'épreuve de ses données contemporaines la validité de concepts forgés par les anthropologues qui ont analysé les rites funéraires ainsi que les relations entre les vivants et les morts dans des sociétés où l'on croyait majoritairement à un monde surnaturel. Par exemple, dans l'Europe chrétienne sur laquelle j'ai mené de longues recherches (Fine, 1994), les morts étaient censés subir une série d'épreuves sur le long chemin les amenant définitivement au repos. Les vivants de leur parenté devaient les aider à franchir ces étapes, en accomplissant une série de gestes et de rites, échelonnés dans le temps. Un temps marqué pour les morts comme pour les vivants par une phase de séparation, une phase liminaire, précédant celle de l'intégration³ ${ }^{3}$ Le temps liminaire, homologue pour les pre-

2 Sur le processus de naturalisation en Suisse comme rite de passage, voir les travaux des anthropologues suisses, Centlivres, 1990 ; Centilivres-Demont, Ossipow, 1991 ; Centlivres, 2000.

3 Je reste très admirative de l'invention en 1909 de la notion de rite de passage de van Gennep dont 
miers et les seconds, dépendait de l'âge du mort, de son statut et des circonstances de son décès". Mais il était transitoire et provisoire. Peut-on parler de "liminalité » durable ou « cristallisée » pour qualifier la situation des parents « deuilleurs » après la mort de leur enfant dans notre société contemporaine? Ces derniers seraient durablement dans cet état en raison de la difficulté à faire face à un statut nouveau (celui de parent privé d'enfant), et parce que leur entourage les confinerait dans une forme d'isolement. Mais les interlocuteurs de l'auteure sont très probablement des parents qui ont développé de nouvelles sociabilités avec des personnes vivant la même expérience : c'est le cas de ce père qui a créé un atelier d'activités manuelles avec d'autres pères endeuillés pour se réconforter mutuellement sans avoir à échanger des mots sur leur souffrance. Ils ont donc un point commun : passé «l'état second » provoqué par l'événement traumatique dont ils disent être sortis (au bout de quelques mois, précise une interlocutrice), leur expérience douloureuse les a conduits à donner un nouveau sens à leur existence par un ensemble de pratiques mémorielles. Celles-ci ne tendraient-elles pas à les maintenir dans un relatif isolement ? Ne se mettraient-ils pas eux-mêmes en marge ? Et si leurs proches les fuient, est-ce par peur de la « contagion » de la mort ? Bien que le terme ait été employé par un des enquêtés, il ne me semble pas recouvrir la même réalité que celle à laquelle les anthropologues font référence pour des sociétés où la contagion de la mort est associée à l'idée de souillure ou de pollution. Ici le terme exprime manifestement la grande peur de la mort que l'on répugne à côtoyer et même à évoquer dans notre société contemporaine.

\section{Les marges comme outil analytique dans le champ de la filiation}

Les deux derniers textes portant sur la filiation enrichissent un champ de recherches que j'ai particulièrement exploré. Après avoir analysé les fonctions symboliques du parrainage dans la longue durée de l'Europe chrétienne, puis sa spécificité par rapport à une autre forme de filiation élective, l'adoption (Fine, 1999a), je me suis intéressée à l'adoption des enfants dans les sociétés contemporaines. L'étude de l'adoption informelle ou sous forme de contrat devant notaire qui n'organisait pas de changement de filiation dans la France ancienne (Fine, 1998), ainsi que celle des formes de l'adoption des enfants lorsque celle-ci devient légale après la Première Guerre mondiale - à savoir substitution de la filiation adoptive à la filiation d'origine, effacement des parents de sang, et même secret de l'adoption pendant plusieurs décennies -, m'ont permis de mettre en évidence les normes dominantes en matière de filiation. Cela d'autant plus facilement que les pratiques adoptives de nos sociétés contrastaient avec celles des mondes africain et océanien déjà bien étudiés par les ethnologues.

Par la suite, j’ai rapproché les pratiques adoptives de nos sociétés d'une autre invention occidentale moderne apparue plus tardivement et s'adressant également aux couples en mal d'enfant : le recours à une insémination avec un donneur anonyme.

la validité me semble toujours d'actualité même si elle a été depuis discutée et enrichie.

${ }^{4}$ Voir la partie intitulée « Entre vivants et morts » (Fine, 1994 : 225-328). 
Dans ce cas, la loi suivant en cela les recommandations des CECOS (Centres d'étude et de conservation des œufs et du sperme humains) permettait la constitution d'une famille sur le modèle de la procréation, en éliminant (par le secret et l'anonymat) le donneur de sperme. L'important étant que l'enfant soit doté de deux parents, selon le modèle procréatif, pas un de plus et de préférence pas un de moins. Là encore l'organisation de cette pratique marginale confortait les analyses précédentes relatives à l'adoption.

Ces arrangements en matière de filiation ont été ébranlés par l'irruption sur la scène sociale des personnes adoptées ou nées d'un donneur anonyme en quête de connaissance sur leurs parents d'origine ou sur leur géniteur. D'autre part, les recompositions familiales après divorce tendaient à faire émerger une figure parentale, celle des beaux-parents dont les liens, très divers avec les enfants, ont fait l'objet de recherches qui faisait apparaître une figure parentale nouvelle dont la fonction, relativement à celle des pères et/ou des mères divorcés, était à définir et dont il était possible de connaître les expériences ${ }^{5}$. Ces éléments rapprochés les uns des autres ont attiré mon attention sur un fait croissant relativement neuf dans nos sociétés : l'existence de formes multiples et variées de pluriparentalités dans ces nouvelles configurations familiales (Fine, 2016).

Les familles homoparentales en particulier se présentaient comme un observatoire extraordinaire de ces inventions nouvelles, les pluriparentalités dans leur cas ne pouvant pas et ne cherchant pas à être occultées (Fine, 2006, 2013). En outre, ces familles ont contribué activement à la réflexion pour élaborer leurs propres revendications en matière de droit de la filiation. Les nombreuses recherches menées sur un phénomène très marginal statistiquement ont été d'un grand apport sur le plan empirique, politique et théorique. En cela ma démarche est très comparable à celles que décrivent Irène Théry et Laurence Hérault dans leur entretien quant à leurs propres recherches. Les deux contributions du dossier qui portent sur les frontières établies par le droit dans le domaine de la filiation en sont une illustration, mais elles m'amènent aussi à une réflexion d'ordre épistémologique.

\section{Frontière juridique et filiation}

L'étude de Marie-Lise Fieyre sur les bâtards dans la haute noblesse à la fin du Moyen Âge et celle sur les relations avec le donneur de sperme connu dans les familles lesbo-parentales d'Isabel Coté et al. montrent de manière exemplaire l'intérêt d'étudier le champ de la filiation en l'abordant par ses marges, mais elles soulèvent une autre question. Les deux types de configurations familiales, très minoritaires dans leur société respective, présentent un point commun : dans les deux cas, le droit de la filiation crée une fron-

\footnotetext{
${ }^{5}$ La bibliographie sur ces familles est importante. Voir en particulier Théry, 1992 ; Cadolle, 2000 ; Martial, 2003.

${ }^{6}$ On trouvera dans ce dernier article (en ligne) une analyse rétrospective des usages de cette notion dans mes différentes recherches avec leurs références depuis 1991.
} 
tière qui se veut étanche entre des enfants et leurs géniteurs. Les bâtards nobles sont exclus d'une succession digne du rang social de leur père. Leurs droits en tous points inférieurs à ceux des enfants légitimes les situent au plus bas de la hiérarchie de la progéniture paternelle, y compris les garçons par rapport à leurs demi-sœurs légitimes. De son côté, le donneur de sperme qui a accepté de contribuer à la procréation d'un enfant avec un couple de lesbiennes, est dépourvu du statut juridique de père. Lui en revanche a accepté et parfois désiré cette place marginale.

Pourtant, bien que le géniteur dans ces familles tout comme le bâtard noble n'aient pas de place dans l'ordre de la filiation légitime, ils appartiennent au groupe de parenté. En effet dans la France de la fin du Moyen Âge et du début de l'époque moderne, qu'il soit noble ou non, le père a l'obligation de fournir nourriture et éducation à l'enfant qu'il a contribué à mettre au monde, il ne peut désavouer sa progéniture (Steinberg, 2016, 2018). Les enfants bâtards nobles sont donc bien considérés comme les enfants de leur père, ils appartiennent incontestablement à son groupe de parenté, ils peuvent d'ailleurs être un jour légitimés.

L'appartenance au groupe familial du donneur connu dans les familles lesbo-parentales, est moins évidente. En effet, les auteurs de l'article montrent qu'elle varie en fonction de la volonté des personnes impliquées dans la procréation. Sa place est labile, elle dépend étroitement des interrelations des différents membres de la configuration familiale et elle évolue dans le temps de la vie. Il peut y avoir une élection réciproque qui peut conduire certains donneurs connus à adopter une partie des comportements associés au statut de parent : conseiller, échanger avec l'enfant, tout en refusant toute obligation morale et juridique.

Ainsi, alors que les frontières juridiques semblent découper des groupes de parents clairement identifiés, si l'on s'attache aux pratiques, les limites du groupe de parenté apparaissent plus floues, celui-ci pouvant éventuellement englober des personnes juridiquement extérieures à lui.

L'historien comme l'anthropologue est pourtant amené à analyser comme un tout l'ensemble des personnes appartenant à ces groupes de parenté que le droit scinde en catégories distinctes. Les familles lesbo-parentales le donnent à voir clairement dans la mesure où la frontière juridique est particulièrement contingente. Elle diffère dans le temps et dans l'espace alors que la configuration familiale est la même et qu'elle s'inscrit dans un même système de filiation (cognatique, bilatéral, indifférencié), celui qui caractérise nos sociétés euro-américaines contemporaines. Par exemple, elle passe actuellement en France au sein même du couple de femmes puisque seule la mère qui a accouché est reconnue comme mère, alors qu'au Québec, les deux mères d'intention, celle qui a accouché et sa compagne, sont juridiquement des mères. Le droit français va probablement bientôt inclure aussi la deuxième mère d'intention?

\footnotetext{
7 Le projet de révision des lois françaises relatives à la bioéthique, adopté en première lecture à l'Assemblée nationale en octobre 2019, prévoit un mode d'établissement de la filiation pour les couples de femmes ayant recours à une procréation assistée avec don permettant aux deux mères d'être inscrites sur l'acte de naissance dès la venue au monde de l'enfant.
} 
D'autre part, si le donneur connu n'est pas un parent au sens juridique, n'en a ni les droits ni les obligations, cela ne l'empêche pas, s'il le désire, de partager avec les parents légitimes certaines fonctions associées généralement à la qualité de père : le fait d'avoir contribué à la naissance, transmis peut-être avec ses gènes quelques traits physiques immanquablement commentés en termes de « ressemblances », avoir exprimé de l'attention et de l'affection et en avoir reçu en retour. Ceci peut expliquer qu'il puisse parfois être qualifié de " père » par l'enfant qu'il a aidé à concevoir, l'inverse étant peut-être moins net.

Sur le plan théorique, il me semble que ces deux études de cas confortent la pertinence des analyses sur la filiation proposées par Flávio Tarnovski (2010 : 23-31). Dans sa thèse, il rappelle l'histoire des divergences entre la tradition anthropologique française et anglo-saxonne quant au contenu du concept de filiation. Dans cette dernière, le terme descent réfère à l'appartenance juridico-politico-religieuse de la descendance, tandis que celui de filiation désignerait les relations de l'enfant avec ses père et mère ; les Anglo-saxons distinguent donc deux composantes de la filiation, alors qu'en français, un seul terme, filiation, est censé rendre compte des deux. L'auteur résume les débats suscités par ces divergences, la manière dont ils ont été repris et discutés entre autres par Francis Zimmermann (1993) et Maurice Godelier (2004). Nous ne pouvons ici entrer dans le détail de ces analyses, mais nous pouvons retenir une des conclusions de l'auteur : le concept que nous utilisons couramment en anthropologie est imprécis. En outre F. Tarnovski note qu'il est d'utilisation particulièrement complexe en tant que catégorie d'analyse dans nos sociétés contemporaines dans la mesure où il existe plusieurs définitions de la filiation concurrentes élaborées par d'autres disciplines, en particulier le droit et la psychanalyse. L'ensemble des réflexions de l'auteur l'amène à reconnaître et l'on ne peut qu'être d'accord avec lui, que la filiation pour les anthropologues ne peut pas se réduire à la filiation des juristes et qu'elle recouvre des réalités plus larges.

Ces difficultés conceptuelles expliquent sans doute en partie que les anthropologues aient préféré user de la notion de parentalité pour rendre compte des nouvelles configurations familiales de notre modernité. À la suite d'Esther Goody (1982, 1999), j'ai contribué avec d'autres à affiner l'analyse de la diffraction des diverses fonctions parentales dans les sociétés euro-américaines. Elles peuvent en effet être occupées par des personnes différentes, les unes parents légaux, les autres hors du champ juridique. Peuvent être inclus dans ces nouvelles parentalités, non seulement les parents d'intention (ceux qui ont décidé de la mise au monde) mais aussi le donneur de sperme, la donneuse d'ovocyte, les beaux-parents éducateurs dans les familles recomposées après divorce, a fortiori le donneur de sperme connu, ami et familier de la famille qu'il a contribué à faire exister. L'intérêt d'adopter une acception large du concept (Fine, 2016: dernier paragraphe) qui paraît a priori absurde tant ces fonctions parentales sont différentes les unes des autres est de permettre les comparaisons avec d'autres sociétés que la nôtre. 
Bien d'autres thématiques auraient pu être explorées relatives aux confins de la parenté. Dans leur entretien, I. Théry et L. Hérault en évoquent plusieurs auxquels je souscris volontiers : les confins de la vie, à savoir la naissance ${ }^{8}$ et la mort, la question de la majorité et de la minorité sexuelle, la question du corps pour les personnes « trans ». I. Théry évoque aussi la frontière poreuse entre parenté et amitié que son enquête sur les proches des mourants du sida lui avait permis de découvrir. J'ai moi-même exploré cette porosité : le baptême des enfants, comme d'autres rites les concernant, dans plusieurs régions d'Europe, créait autrefois entre les parents rituels et les parents de sang des relations de compérage, soit une amitié sacrée, à la vie à la mort. Elle est encore efficiente dans de nombreuses sociétés latino-américaines (Fine, 1999b), et si dans nos sociétés modernes le contenu de la relation entre les parrains/marraines des enfants et leurs parents a perdu son caractère sacré, le parrainage a encore le pouvoir de faire des amis des sortes de parents et des parents des amis (Fine, 1997). Peut-être conviendrait-il d'explorer plus avant cette frontière entre parenté et amitié dans nos sociétés modernes, en se fondant sur les récits d'expérience au moment des ruptures biographiques et en s'efforçant d'être attentif aux différences de genre.

\section{Bibliographie}

Cadolle S. (2000), Être parent, être beau-parent. La recomposition de la famille, Paris, Odile Jacob.

Centuivres P. (dir.) (2000), « Rites, seuils, passages », Communications, nº 70, p. 33-44.

Centlivres P. (2010), Adhésion et diversité culturelle des étrangers en Suisse, Genève, Georg éditeur SA.

Centilivres-demont M., Maillard N., Ossipow L. (1991), Une seconde nature. Pluralisme, naturalisation et identité en Suisse Romande et au Tessin, Lausanne, L’Âge d'Homme.

Courduriès J., Fine A. (dir.) (2014), «Introduction », in Homosexualité et parenté, Paris, Armand Colin, p. 13-41.

Fine A. (1994), Parrains, Marraines. La parenté spirituelle en Europe, Paris, Fayard.

Fine A. (1997), « Parrainage, marrainage et relations familiales dans la société française contemporaine », Lien social et Politiques, RIAC, $\mathrm{n}^{\circ} 37$, p. 157-170.

Fine A. (1998), « Le don d'enfant dans l'ancienne France », in A. FInE (dir.), Adoptions. Ethnologie des parentés choisies, Paris, Maison des sciences de l'homme, p. 61-95.

FINE A. (1999a), « Adoption et parrainage dans l'Europe ancienne », in M. CoRBIER (dir.), Adoption et fosterage, Paris, De Boccard, p. 339-354.

Fine A. (1999b), « Compères et commères. L'amitié rituelle dans l'Europe chrétienne », in G. Ravis-Giordani (dir.), Amitiés. Anthropologie et histoire, Aix en Provence, Publications de l'Université de Provence, p. 257-273.

\footnotetext{
${ }^{8}$ Sur ce point, voir Giraud, 2015.
} 
Fine A. (2006), « Pluriparentalités et homoparentalités dans les sociétés occidentales contemporaines », in A.CADOREt, M.Gross, C. MéCARY, B. PerReAu (dir.), Homoparentalités. Approches scientifiques et politiques, Paris, PUF, p. 43-55.

Fine A. (2013), « Avoir deux pères ou deux mères : révolution ou révélation du sens de la filiation? ", in I. THÉRY (dir.), Mariage de même sexe et filiation, Paris, Éditions de l'EHESS, p. 115-128.

Fine A. (2016), « Retour réflexif sur la notion de pluriparentalité », Le carnet de recherche du centre Jacques Berque. En ligne, consulté le 26/11/19. URL : https://cjb.hypotheses. org/137.

FouRnier S. (2003), Enquête sur l'homoparentalité dans la baie de San Francisco, Mémoire de DEA en anthropologie, Université de Toulouse-Le Mirail.

Giraud A.-S. (2015), « Les péri-parents. À la recherche d'un statut spécifique après une mort périnatale », Recherches familiales, $\mathrm{n}^{\circ} 12$, p. 85-97.

Goody E. (1982), Parenthood and Social Reproduction: Fostering and Occupational Roles in West Africa, Cambridge, Cambridge University Press.

Goody E. (1999), "Sharing and Transferring Components of Parenthood: The West African Case », in M. CoRbier (dir.), Adoption et Fosterage, Paris, De Boccard, p. 369388.

Godelier M. (2004), Les métamorphoses de la parenté, Paris, Fayard.

Martial A. (2000), «L'adoption de l'enfant du conjoint dans les familles recomposées après divorce », in A. Fine et C. NeIRINCK (dir.), Parents de sang, parents adoptifs, Approches juridique et anthropologique de l'adoption. France, Europe, USA, Canada, Paris, LGDJ, p. 189-210.

Martial A. (2003), S'apparenter. Ethnologie des liens de familles recomposées, Paris, Éditions de la Maison des Sciences de l'Homme.

Martial A. (2016), Genre et pluriparentalités dans les nouvelles trajectoires familiales : un parcours de recherche, mémoire d'habilitation à diriger des recherches, à paraître.

Steinberg S. (2016), Une tache au front, Paris, Albin Michel.

Steinberg S. (2018), « Sang des bâtards, sang de la noblesse : à propos des "contextes durables" en anthropologie historique de la parenté », L'Atelier du Centre de recherches historiques, $\mathrm{n}^{\circ}$ 19bis. En ligne, consulté le 26/11/19. URL : http://journals.openedition. org/acrh/8621.

TARNOVSKI F. (2010), Etre père et homosexuel dans la France contemporaine, thèse de doctorat en anthropologie, Toulouse, EHESS.

Thery I., Dhavernas M.-J. (1992), « Le beau-parent dans les familles recomposées. Statut familial, statut social, statut juridique ", Revue des politiques sociales et familiales, $n^{\circ} 27$, p. 37-55.

Zimmermann F. (1993), Enquête sur la parenté, Paris, PUF. 